\title{
The influence of exposure time on color discrimination
}

MICHAEL H. SIEGEL, DEPARTMENT OF PSYCHOLOGY, RIPON COLLEGE, Ripon, Wisc. 54971

An experiment was performed to determine the influence of exposure duration on color discrimination. The current study substantiated earlier research results and established that changes in fixation are responsible for discrimination scores improving at longer exposures.

In an earlier report, the effects of stimulus exposure time on color discrimination ability were studied (Siegel, 1965). The study was conducted to relate the reasonably large amount of information available about the so called wavelength discrimination curve to that concerning temporal relations, both in photochemical reactions and in perception.

Color discrimination was tested at six different exposure times ranging from .02 to $5 \mathrm{sec}$. Despite expectations that sensitivity would reach a maximum value somewhere in the middle of the range, discrimination ability continued to improve as exposure time increased. If adaptation to the stimulus occurred, color differences would have been reduced and observers' discrimination would have deteriorated. Two possible factors accounted for these unexpected results. Because the stimulus was often painfully long and because the only device used to insure invariant fixation was a head and chin rest, it is possible and even likely that observers made several fixations per stimulus exposure. This would effectively shorten the exposure time and might totally erase the effects of adaptztion.

The nature of the observational task permitted another type of error. Although observers were cautioned to base their judgments on the appearance of the stimulus field just before the exposure ended, it is altogether possible that they did not. If the observer responded to the initial color appearance, once again the effective exposure time would have been reduced.

The present study attempted to separate the adapting stimulus from the stimulus to be judged so that the error of judgment described above would not occur. If results continued to demonstrate progressive improvement at longer durations, presumably fixation change would be responsible. Method

A Farrand monochromator with a $1000 \mathrm{~mm}$ focal length presented the stimulus. A specially constructed shutter system previously described (Siegel \& Howard, 1967) permitted presentation of two separate stimuli. The observer was seated in a separate cubicle from which he viewed a white surround continuously illuminated to $.2 \mathrm{ft}-\mathrm{L}$. The circular stimulus appeared through a hole in the surround. The upper half of the horizontally divided 2 deg field served as a standard stimulus. As in the previous experiment, it was set to a wavelength of $575 \mathrm{~m} \mu$ at a bandwidth of $5 \mathrm{~m} \mu$ and a luminance of $.2 \mathrm{ft}-\mathrm{L}$ for the entire experiment. The lower half of the field served as the variable stimulus.

Before each experimental session a pretest was performed to allow the observer to equate the brightness of each of the variable stimuli to that of the standard. The method of constant stimulus differences was used. The stimulus range consisted of five steps, each of which was presented 10 times in a random order. A session consisted of two separate measurements: one for wavelengths longer than the standard during which the observer was asked whether or not the variable stimulus was yellower than the standard; and the other for wavelengths shorter than the standard during which the observer was asked whether or not the variable stimulus was greener than the standard. Each stimulus consisted of a pre-exposure or adaptation flash and a second flash which was designated as the exposure. Between these two flashes the field

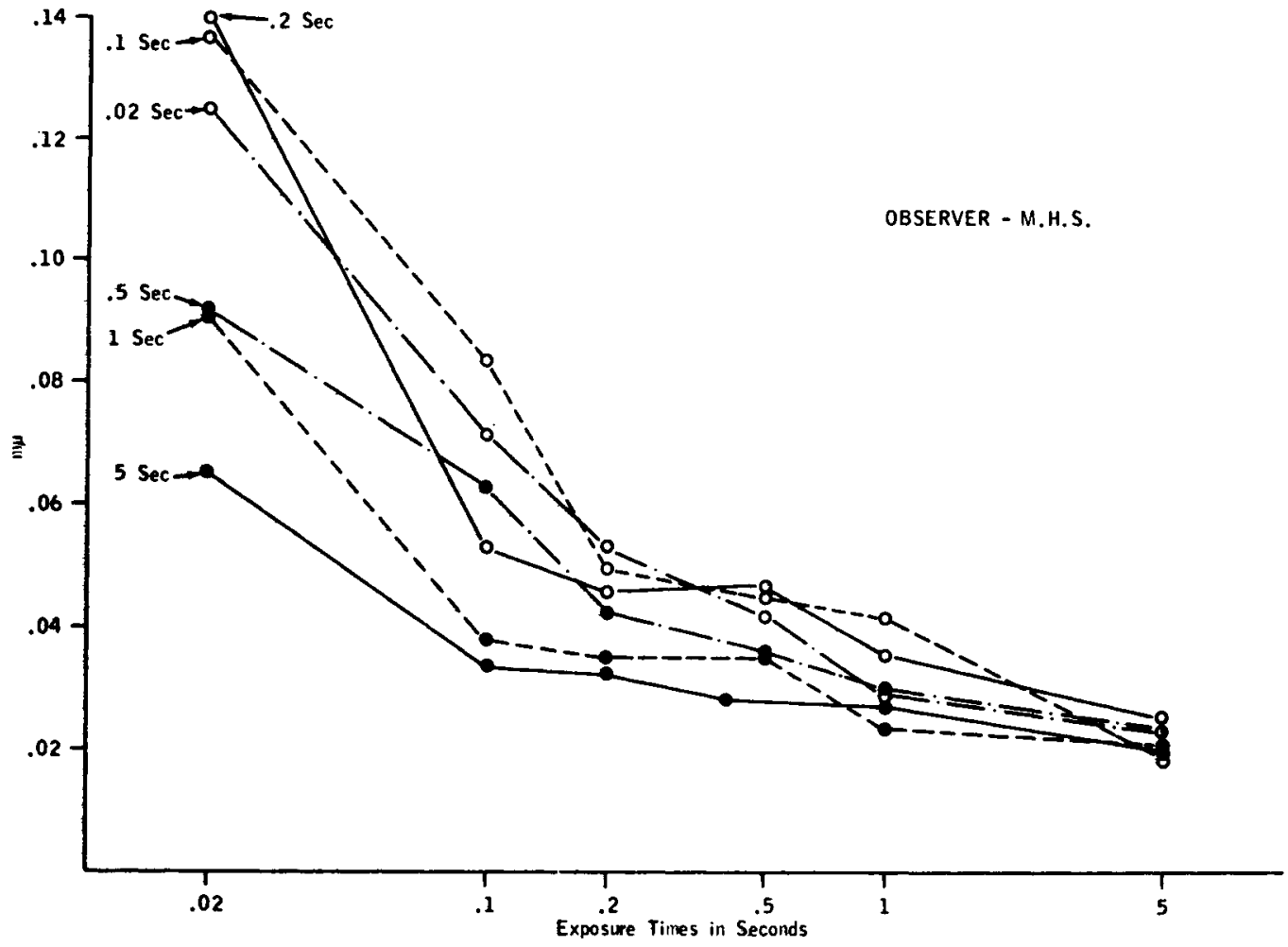

Fig. 1. Sensitivity as a function of exposure time. 


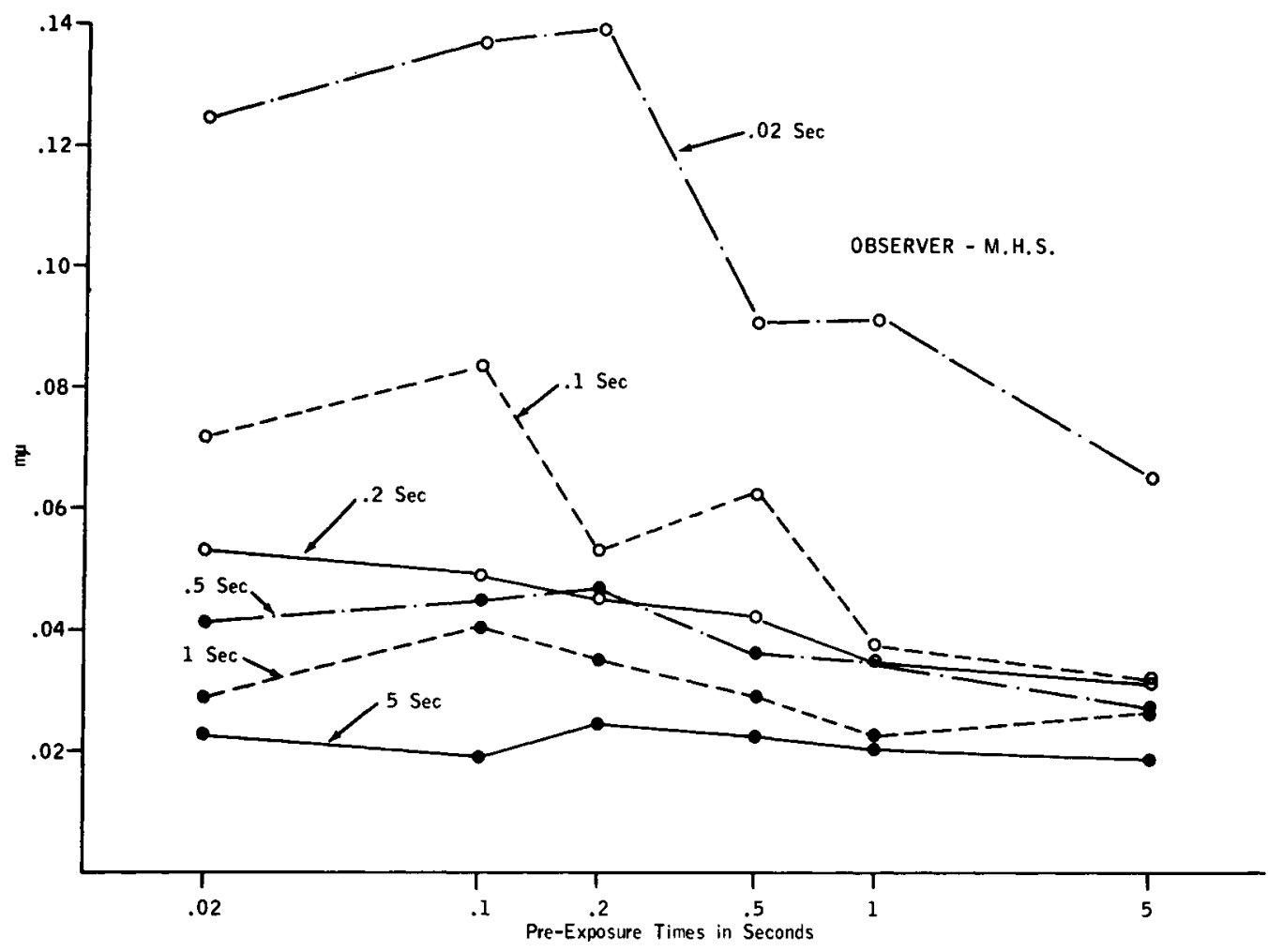

Fig. 2. Sensitivity as a function of pre-exposure time.

was dark for $1.5 \mathrm{sec}$. Both the standard and the variable halves appeared and disappeared together.

The experimental design called for six different exposure times: $.02 ; .1 ; .2 ; .5 ; 1 ;$ and $5 \mathrm{sec}$. The same six times were used for the pre-exposure, giving a total of 36 pre-exposure-exposure combinations. One experimental session was run at each of these 36 conditions and the order in which the conditions appeared was reversed and a second experimental session was run at each condition.

Data were collected from three observers who were thoroughly familiar with the sort of discrimination required. Each used only his right eye. A head and chin rest was employed to minimize head movements, but there were no controls for changes in the observers' fixations.

\section{Results}

The measure of sensitivity used was the normalized standard deviation of the observers' judgments. Figure 1 presents the experimental results for observer MHS. Since the results for the three observers are virtually identical, data are presented only for one. As in the previous experiment, it is obvious that there is a trend toward better discrimination scores at longer exposures. Indeed, as previously reported. even at the longest experimental duration, discrimination ability continued to improve. Figure 2 recasts the data by showing sensitivity as a function of pre-exposure time for the same observer. There is almost no relation between the duration of the pre-exposure flash and measured sensitivity. One exception may be found in the data at very brief exposure durations. Both at .02 and at $.1 \mathrm{sec}$ there is some, though not systematic, improvement in sensitivity as pre-exposure time is increased. It seems safe to conclude that observers were influenced by the appearance of the pre-exposure flash to a greater extent in the very brief exposures than they were in longer exposures. In any event, except for these very brief exposures, pre-exposure flash did not influence observers' judgments. The improvement in measured discrimination as a function of the increasing exposure time both in the present experiment and in the previously reported one must therefore result from fixation change. Since it is not possible to eliminate eye movement and retain a stimulus for very long, it is necessary to acknowledge the fact that observers do indeed move their heads and eyes during their observations. Therefore, when sensitivity data are reported, just as complete and meticulous attention should be given to specifying the experimental conditions and duration as is typically given for most other relevant stimulus properties.

\section{REFERENCES}

SIEGEL, M. H. Color discrimination as a function of exposure time. J. Opt. Soc. Amer., 1965, 55, 566-568.

SIEGEL, M. H., \& HOWARD, E. An accurate, adjustable double shutter. Percept. Mot. Skills, 1967, 24, 673-674. 